\title{
THE HELICAL TRANSFORM AS A CONNECTION BETWEEN ERGODIC THEORY AND HARMONIC ANALYSIS
}

\author{
IDRIS ASSANI AND KAKL PETERSEN
}

\begin{abstract}
Direct proofs are given for the formal equivalence of the $L^{2}$ boundedness of the maximal operators corresponding to the partial sums of Fourier series, the range of a discrete helical walk, partial Fourier coefficients, and the discrete helical transform. Strong $(2,2)$ for the double maximal (ergodic) helical transform is extended to actions of $\mathbb{R}^{d}$ and $\mathbb{Z}^{d}$. It is also noted that the spectral measure of a measure-preserving flow has a continuity property at $\infty$, the Local Ergodic Theorem satisfies a Wiener-Wintner property, and the maximal helical transform is not weak $(1,1)$.
\end{abstract}

\section{INTRODUCTION}

In [1] the Carleson-Hunt Theorem on the maxima of the partial sums of the Fourier series of an $L^{2}$ function was used to prove a continuity property of the spectral measures of measure-preserving transformations, strengthening Gaposhkin's necessary and sufficient spectral condition for almost everywhere convergence of the Cesàro averages of the powers of a normal operator. The proof depended on computations involving the "rotated ergodic Hilbert transform"

$$
H_{\theta} f(x)=\lim _{n \rightarrow \infty} \sum_{k=-n}^{n} \frac{e^{i k \theta} f\left(T^{k} x\right)}{k}
$$

(the ${ }^{\prime}$ on the sum means that the term with 0 denominator is omitted), which we call here the helical transform of $f \in L^{1}$, and on a harmonic analysis maximal lemma concerning the sequence of maximal partial Fourier coefficients of an $L^{2}$ function on the interval. It was this latter lemma that was proved from the Carleson-Hunt Theorem, by means of some transference arguments and subharmonicity. This lemma is in fact equivalent to the Carleson-Hunt Theorem, as is a discrete formulation, which may be amenable to combinatorial or geometrical analysis, that arises from applying it to step functions (see [10] for an earlier version). This new discrete formulation we call the case of helical walks, since it has to do with the mean square of the maximal distances from the origin achievable by a walker who takes steps of a predetermined size, between

Received by the editors January 25, 1990.

1980 Mathematics Subject Classification (1985 Revision). Primary 28D05; Secondary 42A20, 42A50, 47A60.

Key words and phrases. Carleson-Hunt Theorem, Hilbert transform, Local Ergodic Theorem, Wiener-Wintner property, maximal inequality, large sieve, spectral measure. 
steps turning through a fixed fraction of the circle, and stopping whenever he chooses, before setting out on another foray with another fixed turning angle.

Our first purpose is to provide extremely direct and simple proofs for the implications among these results, thereby clarifying the relationships among them. This is carried out in $\S 2$, where we begin by using a clever argument from the work of Davenport and Halberstam on the large sieve to show how the Carleson-Hunt Theorem directly implies (without having to use transference or subharmonicity) the estimate on helical walks and therefore also the maximal lemma for partial Fourier coefficients. As in [1], the equivalence with the boundedness of the maximal helical transform on $l^{2}$ is then immediate. The arguments in the other direction are even simpler. A thorough understanding of the connections among these results extends their range of applicability and may lead eventually to a simpler proof of all of them.

The main ergodic-theoretic result in [1] was a weak $(2,2)$ estimate for the double maximal function for the helical transform, the supremum being taken over both $\theta$ and $n$. In $\S 3$ we extend this result to the case of actions of $\mathbb{R}^{d}$ and $\mathbb{Z}^{d}$. Of course higher-dimensional analogues of the other equivalent results mentioned in $\S 1$ hold as well. We can use this result for an action of $\mathbb{R}$ to show that the spectral measure of a measure-preserving flow has a type of continuity at $\infty$; this strengthens, for a measure-preserving flow, Gaposhkin's necessary and sufficient condition for a flow of normal operators to satisfy the Local Ergodic Theorem. In this connection, we note that the Local Ergodic Theorem satisfies a Wiener-Wintner property: multiplying inside the integral by $e^{2 \pi i s \theta}$, one obtains convergence off a set of measure 0 which does not depend on $\theta$. While we have made some progress towards the proof of the analogous statement for the helical transform, the result is not yet in hand. Finally, a few remarks about $L^{p}$ for $p \neq 2$ : the double maximal helical transform is not weak $(1,1)$; and for $p>1, p \neq 2$, many of the analogous statements to the foregoing hold, so long as one allows correctly for the ways that duality and interpolation function in these cases.

It is a pleasure to acknowledge the helpful suggestions that we have obtained from Pascal Auscher, James Campbell, John Chalk, and Michael Lacey. The second author also thanks the Indian Statistical Institute, Calcutta, for hospitality during part of this work and the National Science Foundation for support through grants DMS-8620132 and DMS-8900136.

\section{Equivalents of the Carleson-Hunt Theorem}

We will show how to derive the four following statements involving strong type $(2,2)$ estimates quickly from one another. As usual, $C$ denotes a constant that is not necessarily the same from one appearance to the next.

(1) Partial sums of Fourier series (Carleson-Hunt $[3,7])$. For $\hat{h} \in l^{2}(\mathbb{Z})$ and $x \in[0,1)$, let

$$
S^{*} \hat{h}(x)=\sup _{n>0}\left|\sum_{j=-n}^{n} \hat{h}(j) e^{2 \pi i j x}\right| .
$$

Then there is a constant $C$ such that $\left\|S^{*} \hat{h}\right\|_{L^{2}[0,1)} \leq C\|\hat{h}\|_{l^{2}(\mathbb{Z})}$ for all $\hat{h} \in l^{2}(\mathbb{Z})$.

(2) Helical walks. For $N=1,2, \ldots$ and $r=1, \ldots, N$ let $\omega_{r}=e^{2 \pi i r / N}$. Then there is a constant $C$ such that for any $N$, any $v_{1}, \ldots, v_{N} \geq 0$ and 
any choice of $N_{r}=1, \ldots, N$ for $r=1, \ldots, N$,

$$
\frac{1}{N} \sum_{r=1}^{N}\left|\sum_{m=1}^{N_{r}} v_{m} \omega_{r}^{m}\right|^{2} \leq C \sum_{m=1}^{N} v_{m}^{2} .
$$

(3) Partial Fourier coefficients [1]. For $h \in L^{2}[0,1)$ and $j \in \mathbb{Z}$, let

$$
I^{*} h(j)=\sup _{\varepsilon>0}\left|\int_{0}^{\varepsilon} h(x) e^{-2 \pi i j x} d x\right| .
$$

Then there is a constant $C$ such that $\left\|I^{*} h\right\|_{l^{2}(\mathbb{Z})} \leq C\|h\|_{L^{2}[0,1)}$ for all $h \in$ $L^{2}[0,1)$.

(4) Maximal helical transform on $l^{2}$. For $a \in l^{2}(\mathbb{Z})$ and $\theta \in \mathbb{R}$ define

$$
H_{\theta} a(j)=\sum_{k=-\infty}^{\infty} \frac{e^{2 \pi i(j-k) \theta} a_{j-k}}{k} \text { and } H^{*} a(j)=\sup _{\theta \in \mathbb{R}}\left|H_{\theta} a(j)\right| .
$$

Then there is a constant $C$ such that for all $a \in l^{2}(\mathbb{Z}),\left\|H^{*} a\right\|_{l^{2}} \leq C\|a\|_{l^{2}}$.

(1) $\Rightarrow(2)$ We extract part of an argument used by Davenport and Haberstam [4] to improve some estimates by Roth and Bombieri on the large sieve. Fix $N=1,2, \ldots$, let $\alpha=1 /(2 N)$, and let

$$
\psi(x)=\sum_{n=-\infty}^{\infty}\left[\frac{\sin (n \pi \alpha)}{n \pi \alpha}\right]^{2} e^{2 \pi i n x} .
$$

This function is chosen because its sequence of Fourier coefficients has some very useful properties. If $\gamma_{\alpha}=\left(\chi_{[1-\alpha / 2,1]}+\chi_{[0, \alpha / 2]}\right) / \alpha$, then $\psi=\gamma_{\alpha} * \gamma_{\alpha}$, so it follows readily that

$$
\psi(x)= \begin{cases}\frac{1}{\alpha}\left(1-\frac{\|x\|}{\alpha}\right) & \text { if }\|x\| \leq \alpha, \\ 0 & \text { if }\|x\| \geq \alpha,\end{cases}
$$

(where $\|x\|=d(x, \mathbb{Z})$ ); and if $b_{n}=[\sin (n \pi \alpha) /(n \pi \alpha)]^{2}$ for $n \neq 0, b_{0}=1$, then

$$
\int_{0}^{1} \psi^{2}(x) d x=\sum_{n=-\infty}^{\infty} b_{n}^{2}=\frac{2}{3 \alpha}
$$

and

$$
b_{n}^{-2} \leq b_{N}^{-2} \leq\left(\frac{\pi}{2}\right)^{2} \quad \text { for } n \leq N .
$$

Let $x_{r}=r / N$ for $r=1, \ldots, N$, choose any $N_{r}=1, \ldots, N$, and let

$$
\tau_{r}(x)=\sum_{m=1}^{N_{r}} \frac{v_{m}}{b_{m}} e^{2 \pi i m x}
$$

Then

$$
S_{r}(x)=\sum_{m=1}^{N_{r}} v_{m} e^{2 \pi i m x}=\psi * \tau_{r}(x)=\int_{-\alpha}^{\alpha} \psi(y) \tau_{r}(x-y) d y,
$$

so that by Hölder's inequality

$$
\left|s_{r}(x)\right|^{2} \leq \int_{-\alpha}^{\alpha} \psi^{2}(y) d y \int_{-\alpha}^{\alpha}\left|\tau_{r}(x-y)\right|^{2} d y \leq \frac{4 N}{3} \int_{x-\alpha}^{x+\alpha}\left|\tau_{r}(y)\right|^{2} d y,
$$


and hence,

$$
\left|s_{r}\left(x_{r}\right)\right|^{2} \leq \frac{4 N}{3} \int_{x_{r}-\alpha}^{x_{r}+\alpha}\left|\tau_{r}(y)\right|^{2} d y \leq \frac{4 N}{3} \int_{x_{r}-\alpha}^{x_{r}+\alpha} \sup _{r \leq N}\left|\tau_{r}(y)\right|^{2} d y .
$$

Therefore, using the fact that the intervals $\left(x_{r}-\alpha, x_{r}+\alpha\right)$ are disjoint (the points $x_{r}$ are "well spaced," in the sense of Davenport and Halberstam) and (1),

$$
\begin{aligned}
\sum_{r=1}^{N}\left|s_{r}\left(x_{r}\right)\right|^{2} & \leq \frac{4 N}{3} \int_{0}^{1} \sup _{r \leq N}\left|\tau_{r}(y)\right|^{2} d y \leq \frac{4 N}{3} C \sum_{m=1}^{N} \frac{v_{m}^{2}}{b_{m}^{2}} \\
& \leq \frac{4 N}{3} C\left(\frac{\pi}{2}\right)^{2} \sum_{m=1}^{N} v_{m}^{2} .
\end{aligned}
$$

(2) $\Rightarrow$ (3) We may assume that $h$ is a nonnegative step function, taking the value $v_{m}$ on the interval $[(m-1) / N, m / N), m=1, \ldots, N$. For each $j \in \mathbb{Z}$ and any choice of $\varepsilon_{j}=\left(N_{j} / N\right)+\delta_{j}\left(N_{j}=0, \ldots, N, 0 \leq \delta_{j}<1 / N\right)$,

(A) $\int_{0}^{\varepsilon_{j}} h(x) e^{-2 \pi i j x} d x=\sum_{m=1}^{N_{j}} v_{m} \int_{\frac{m-1}{N}}^{\frac{m}{N}} e^{-2 \pi i j x} d x+v_{N_{j}+1} \int_{N_{j} / N}^{N_{j} / N+\delta_{j}} e^{-2 \pi i j x} d x$.

If $v^{*}=\max _{m} v_{m}$, then the second of these terms may be estimated by

$$
C \frac{v^{*}}{|j|}\left|e^{-2 \pi i N_{j} / N}\left(e^{-2 \pi i j \delta_{j}}-1\right)\right| \leq \begin{cases}C \frac{v^{*}}{|j|} \frac{|j|}{N} & \text { if }|j| \leq N \\ C \frac{v^{*}}{|j|} & \text { if }|j|>N\end{cases}
$$

so that this term contributes to $\left\|l^{*} h\right\|_{l^{2}(\mathbb{Z})}^{2}$ at most

$$
C\left[\frac{v^{*}}{N}\right]^{2} \cdot N+C v^{* 2} \sum_{|j|>N} \frac{1}{j^{2}}=C \frac{v^{* 2}}{N} \leq C\|h\|_{2}^{2} .
$$

Suppose that $\varepsilon_{j}$ is chosen for each $j$ so as to maximize the first of the two terms in (A), which may be written as

$$
\frac{-1}{2 \pi i j} \sum_{m=1}^{N_{j}} v_{m} e^{-2 \pi i j(m-1) / N}\left(e^{-2 \pi i j / N}-1\right) \text {. }
$$

Evidently the maximizing choice of $N_{j}$ depends only on the congruence class of $j \bmod N$; thus we write $j=k N+r$ with $1 \leq r \leq N$ and $k \in \mathbb{Z}$ and replace $N_{j}$ by $N_{r}$. The contribution of this term to $\left\|I^{*} h\right\|_{l^{2}(\mathbb{Z})}^{2}$ is therefore at most

$$
\begin{aligned}
C \sum_{k=-\infty}^{\infty} \sum_{r=1}^{N} \frac{1}{(k N+r)^{2}}\left|\sum_{m=1}^{N_{r}} v_{m} \omega_{r}^{m-1}\left(\omega_{r}-1\right)\right|^{2} \\
=C \sum_{r=1}^{N} \frac{1}{r^{2}}\left|\left(\omega_{r}-1\right) \sum_{m=1}^{N_{r}} v_{m} \omega_{r}^{m-1}\right|^{2} \\
\quad+\frac{1}{N^{2}} \sum_{k \neq 0} \sum_{r=1}^{N} \frac{1}{(k+r / N)^{2}}\left|\left(\omega_{r}-1\right) \sum_{m=1}^{N_{r}} v_{m} \omega_{r}^{m-1}\right|^{2} .
\end{aligned}
$$


Using $\left|\omega_{r}-1\right| / r \leq C / N$ on the first of these terms and summing on $k$ and using (2) on the second, this is bounded by

$$
\frac{C}{N^{2}} \sum_{r=1}^{N}\left|\sum_{m=1}^{N_{r}} v_{m} \omega_{r}^{m-1}\right|^{2}+C \frac{1}{N} \sum_{m=1}^{N} v_{m}^{2} \leq C \frac{1}{N} \sum_{m=1}^{N} v_{m}^{2}=C\|h\|_{L^{2}[0,1)}^{2} .
$$

(3) $\Rightarrow$ (2) Apply (3) to step functions as above and use the fact that $\left|\omega_{r}-1\right| / r \geq C / N$ for $-N / 2 \leq r \leq N / 2$.

(3) $\Leftrightarrow(4)$ Let $h(x)=\sum_{k=-\infty}^{\infty} a_{k} e^{2 \pi i k x}$. Then for all $j \in \mathbb{Z}$,

$$
\int_{0}^{\varepsilon} h(x) e^{-2 \pi i j x} d x=a_{j} \varepsilon+\sum_{k=-\infty}^{\infty} \frac{e^{2 \pi i(k-j) \varepsilon} a_{k}}{k-j}-\sum_{k=-\infty}^{\prime} \frac{a_{k}}{k-j} .
$$

Since the discrete Hilbert transform $\sum_{k=-\infty}^{\infty} a_{k} /(k-j)$ is known to be strong $(2,2)$ (easily seen by applying the Fourier transform), the result follows immediately.

$(2) \Rightarrow(1)$ For $\hat{h} \in l^{2}(\mathbb{Z}), K \geq 1$, and $x \in[0,1)$, let

$$
S_{K}^{*} \hat{h}(x)=\sup _{1 \leq n \leq K}\left|\sum_{j=-n}^{n} \hat{h}(j) e^{2 \pi i j x}\right|=\left|\sum_{j=-n(x)}^{n(x)} \hat{h}(j) e^{2 \pi i j x}\right| .
$$

Since this is a continuous function of $x$, we may choose $N \geq K$ large enough that

$$
\int_{0}^{1}\left|S_{K}^{*} \hat{h}(x)\right|^{2} d x
$$

is arbitrarily well approximated by a Riemann sum

$$
\sum_{r=1}^{N}\left|\sum_{j=-N_{r}}^{N_{r}} \hat{h}(j) \omega_{r}^{j}\right|^{2} \cdot \frac{1}{N}
$$

Applying (2) twice, to the negative and nonnegative ranges of $j$, this is bounded by

$$
C \sum_{j=-N}^{N}|\hat{h}(j)|^{2} \leq C\|h\|_{2}^{2}
$$

To be able to transfer estimates of this kind to the ergodic setting, it is necessary to estimate a double maximal function, in which the supremum is taken over two parameters. This was noted in [1], where a weak type $(2,2)$ estimate was proved for the double maximal helical transform on $l^{2}$, and strong type $(2,2)$ was conjectured. It turns out that strong type $(2,2)$ does indeed follow from (4) by a method routine in harmonic analysis; we thank an earlier referee for pointing this out. The application of this method to this particular situation involves a couple of small but tricky details, so we sketch one way in which it can be done.

5. Corollary (Strong $(2,2)$ for the double maximal helical transform on $l^{2}$ ). For $a \in l^{2}(\mathbb{Z})$ and $\theta \in \mathbb{R}$ define

$$
H^{* *} a(j)=\sup _{\substack{\theta \in \mathbb{R} \\ n>0}}\left|\sum_{k=-n}^{n} \frac{e^{2 \pi i(j-k) \theta} a_{j-k}}{k}\right| .
$$


Then there is a constant $C$ such that for all $a \in l^{2}(\mathbb{Z})$,

$$
\left\|H^{* *} a\right\|_{l^{2}(\mathbb{Z})} \leq C\|a\|_{l^{2}(\mathbb{Z})} \text {. }
$$

Proof. Define $a^{\varepsilon}(j)=e^{2 \pi i j \varepsilon} a(j)$, and for a function $u$ let $D_{N} u(t)=\frac{1}{N} u\left(\frac{t}{N}\right)$. Define

$$
\begin{array}{ll}
k_{1}(t)=\frac{1}{t} \chi_{\{|t| \geq 1\}}(t), & k_{N}(t)=\frac{1}{t} \chi_{\{|t| \geq N\}}(t)=D_{N} k_{1}(t), \\
k(t)=\frac{1}{t} \quad \text { for } t \neq 0, & k(0)=0 .
\end{array}
$$

Convolutions with $k$ are defined by the usual symmetric singular integrals. Let $\phi$ be a smooth function on $\mathbb{R}$ with compact support and total integral 1 . For a sequence $u(n)$, define $\bar{u}(t)=u\langle t\rangle$ for $t \in \mathbb{R}$, where $\langle t\rangle$ denotes the nearest integer to $t$. Then $\bar{u} *_{\mathbb{R}} \bar{v}(j)=u *_{\mathbb{Z}} v(j)$ for $j \in \mathbb{Z}$, and $\|\bar{u}\|_{L^{2}(\mathbb{R})}=$ $\|u\|_{l^{2}(\mathbb{Z})}$.

We want to show that $\sup _{N, \varepsilon}\left|a^{\varepsilon} *_{\mathbb{L}} k_{N}(j)\right|$ is strong $(2,2)$. Now $k_{N}-\bar{k}_{N}$ is integrable on $\mathbb{R}$, with $L^{1}$ norm independent of $N$. Therefore, by Minkowski's inequality, it is enough for us to show that $\sup _{N, \varepsilon}\left|\bar{a}^{\varepsilon} *_{\mathbb{R}} k_{N}(j)\right|$ has $l^{2}$ norm bounded by a constant times the $l^{2}$ norm of $a$. Since $D_{N}(k * \phi)=k * D_{N} \phi$, we may write

$$
\bar{a}^{\varepsilon} * k_{N}=\bar{a}^{\varepsilon} * k * D_{N} \phi+\bar{a}^{\varepsilon} * D_{N}\left(k_{1}-k * \phi\right) .
$$

As in [11, p. 67], from the smoothness of $\phi$ it follows that $k_{1}-k * \phi$ is bounded, as well as integrable and monotonic for $|x| \geq 2$. Therefore we may find even integrable functions $\psi$ and $\eta$ which are decreasing functions of $|x|$ and such that $\psi \geq|\phi|$ and $\eta \geq\left|k_{1}-k * \phi\right|$. (The sizes of $\psi$ and $\eta$, and hence the bounding constants obtained in this way, will depend on $\phi$ and its derivative.) Then we may apply the homogeneity and dyadic decomposition argument found, for example, in [12, pp. 83-84]: the second term is dominated by

$$
\begin{aligned}
\mid \bar{a}^{\varepsilon} * & D_{N}\left(k_{1}-k_{1} * \phi\right)|(j) \leq| \bar{a}|*| D_{N} \psi \mid(j) \\
= & \sum_{r=0}^{\infty} \int_{2^{r} \leq|t| \leq 2^{r+1}} D_{N} \psi(t)|\bar{a}(j-t)| d t+\int_{-1}^{1} D_{N} \psi(t)|\bar{a}(j-t)| d t \\
\leq & \sum_{r=0}^{\infty} \frac{1}{N} \psi\left(\frac{2^{r}}{N}\right) \int_{2^{r} \leq|t| \leq 2^{r+1}}|\bar{a}(j-t)| d t \\
& +C\|\psi\|_{1}(|\bar{a}(j-1)|+|\bar{a}(j)|+|\bar{a}(j+1)|) \\
\leq & \sum_{r=0}^{\infty}\left[\frac{1}{2^{r+1}} \int_{2^{r} \leq|t| \leq 2^{r+1}}|\bar{a}(j-1)| d t\right] 2^{r-1} \frac{1}{N} \psi\left(\frac{2^{r}}{N}\right)+C\|\psi\|_{1} M|\bar{a}|(j) \\
\leq & M \bar{a}(j) \sum_{r=0}^{\infty} \int_{2^{r-1} \leq|t| \leq 2^{r}} \frac{1}{N} \psi\left(\frac{t}{N}\right) d t+C\|\psi\|_{1} M|\bar{a}|(j) \\
\leq & M \bar{a}(j) \int_{1 / 2}^{\infty} D_{N} \psi(t) d t+C\|\psi\|_{1} M|\bar{a}|(j) \leq C\|\psi\|_{1} M|\bar{a}|(j) .
\end{aligned}
$$

A similar computation applies to the first term, except that $\bar{a}$ is replaced by $\bar{a} \varepsilon * \bar{k}$. Again because of Minkowski's inequality we may just as well consider $\bar{a}^{\varepsilon} * \bar{k}$. Taking the supremum on $\varepsilon$ then yields $H^{*} a$, so that the estimate after 
the homogeneity argument is $C\|\eta\|_{1} M \overline{H^{*} a}(j)$, which is strong $(2,2)$ because (4) is.

\section{CONSEQUENCES FOR ERGODIC THEORY}

The double maximal inequality for the helical transform transfers to the ergodic-theoretic setting. In [1] the weak $(2,2)$ version of this result was used to prove a continuity property at 0 of the spectral measure of a measurepreserving transformation. Here we extend the double maximal estimate to the case of actions of $\mathbb{R}, \mathbb{R}^{d}$, and $\mathbb{Z}^{d}, d \geq 1$; the inequality for a measurepreserving flow has as a consequence the continuity at $\infty$ of its spectral measure.

6. Theorem (Strong $(2,2)$ for the double maximal helical transform for m.p.t.'s and flows). Let $T: X \rightarrow X$ be a measure-preserving transformation (m.p.t.) and $\left\{T_{s}:-\infty<s<\infty\right\}$ a measure-preserving flow on a measure space $(X, \mathscr{B}, \mu)$. For $f \in L^{2}(X, \mathscr{B}, \mu)$ define

$$
H^{* *} f(x)=\sup _{n, \theta}\left|\sum_{k=-n}^{n} \frac{e^{2 \pi i k \theta} f\left(T^{k} x\right)}{k}\right|
$$

and

$$
F^{* *} f(x)=\sup _{\eta, \theta}\left|\int_{\eta \leq|s| \leq \frac{1}{\eta}} \frac{e^{2 \pi i s \theta} f\left(T_{s} x\right)}{s} d s\right| .
$$

Then there is a constant $C$ such that

$$
\left\|H^{* *} f\right\|_{2} \leq C\|f\|_{2} \text { and }\left\|F^{* *} f\right\|_{2} \leq C\|f\|_{2} \quad \text { for all } f \in L^{2}(X, \mathscr{B}, \mu) .
$$

Proof. Strong $(2,2)$ for $H^{* *}$ follows by means of the usual transference argument found, for example, in [2] and [13]. We apply this estimate to the time $\delta$ map of the flow and a function

$$
f(x)=\int_{-\infty}^{\infty} g\left(T_{s} x\right) \phi(s) d s,
$$

where $g$ is bounded and measurable on $(X, \mathscr{B}, \mu)$ and $\phi$ is an infinitely differentiable function on $\mathbb{R}$ with compact support. For such an $f, f\left(T_{s} x\right)$ is a uniformly continuous function of $s$, uniformly in $x$. Since the set of all such $f$ is dense in $L^{2}$, the result will follow. Let

$$
F_{N}^{* *} f(x)=\sup _{\substack{1 / N \leq \theta \leq N \\ 1 / N \leq \eta \leq 1}}\left|\int_{\eta \leq|s| \leq \frac{1}{\eta}} \frac{e^{2 \pi i s \theta} f\left(T_{s} x\right)}{s} d s\right| .
$$

Fix $N=1,2, \ldots$ and choose $\delta$ so small that for each $x$ and each $\theta \in$ $[1 / N, N]$ the oscillation of $e^{2 \pi i s \theta} f\left(T_{s} x\right) / s$, as a function of $s$, is less than $\|f\|_{2} / N$ on each subinterval of $[1 / N, N]$ of length less than $\delta$. For any $\theta, \eta$ with $1 / N \leq \theta \leq N, 1 / N \leq \eta \leq 1$, define $m, n, \tau$, and $\gamma$ by $\eta=m \delta+$ 
$\tau, 1 / \eta=n \delta+\gamma, 0 \leq \tau, \gamma<\delta$. Write

$$
\begin{aligned}
\int_{\eta \leq|s| \leq \frac{1}{\eta}} \frac{e^{2 \pi i s \theta} f\left(T_{s} x\right)}{s} d s= & \sum_{\substack{k=-n \\
k \notin[-m, m-1]}}^{n} \int_{k \delta}^{(k+1) \delta} \frac{e^{2 \pi i s \theta} f\left(T_{s} x\right)}{s} d s \\
& -\int_{\substack{1 / \eta \leq|s| \leq(n+1) \delta \\
\text { or } m \delta \leq \leq s \mid \leq \eta}} \frac{e^{2 \pi i s \theta} f\left(T_{s} x\right)}{s} d s .
\end{aligned}
$$

The second term is bounded by $2\|f\|_{2}$, while the first may be written as

$$
\sum_{\substack{k=-n \\ k \notin[-m, m-1]}}^{n} \frac{e^{2 \pi i k \delta \theta} f\left(T_{g}^{k} x\right)}{k}+\int_{\eta \leq|s| \leq \frac{1}{\eta}}\left[\frac{e^{2 \pi i s \theta}}{s}-\frac{e^{2 \pi i\left\lfloor\frac{s}{\delta}\right\rfloor \delta \theta} f\left(T_{\delta}^{\left\lfloor\frac{s}{\delta}\right\rfloor} x\right)}{\left\lfloor\frac{s}{\delta}\right\rfloor \delta}\right] d s .
$$

The second of these terms is also bounded by $\|f\|_{2}$, while the first is no more than $2 H^{* *} f$, with respect to the transformation $T_{\delta}$. Therefore $\left\|F_{N}^{* *}\right\|_{2}^{2} \leq$ $C\|f\|_{2}^{2}$ with $C$ independent of $N$, so the result follows.

7. Remark. By a slight modification of the above argument, we can show that also

$$
\sup _{\theta, \eta, \varepsilon}\left|\int_{\eta \leq|s| \leq \varepsilon} \frac{e^{2 \pi i s \theta} f\left(T_{s} x\right)}{s} d s\right|
$$

is strong $(2,2)$.

8. Theorem (Two-dimensional case of strong $(2,2)$ for partial Fourier coefficients). For $h \in L^{2}([0,1) \times[0,1))$ and $(m, n) \in \mathbb{Z}^{2}$, let

$$
I^{*} h(m, n)=\sup _{\varepsilon>0}\left|\int_{0}^{\varepsilon} \int_{0}^{\varepsilon} h(x, y) e^{-2 \pi i(m x+n y)} d x d y\right| .
$$

Then there is a constant $C$ such that $\left\|I^{*} h\right\|_{l^{2}\left(\mathbb{Z}^{2}\right)} \leq C\|h\|_{L^{2}([0,1) \times[0,1))}$ for all $h \in L^{2}([0,1) \times[0,1))$.

Proof. It seems that an attempt simply to apply the corresponding result for a one-dimensional action does not quite work; however, a nice trick involving integrating over sectors, which we found in [5], makes the estimate easy. Thus we consider

$$
I_{1}^{*} h(m, n)=\sup _{\varepsilon<0}\left|\int_{x=0}^{\varepsilon} \int_{y=0}^{x} h(x, y) e^{-2 \pi i(m x+n y)} d y d x\right|,
$$

and a similar $I_{2}^{*} h(m, n)$, for which the roles of $x$ and $y$ are interchanged. If

$$
h_{n}(x)=\int_{y=0}^{x} h(x, y) e^{-2 \pi i n y} d y=\left[h(x, \cdot) \chi_{[0, x]}(\cdot)\right]^{\wedge}(n),
$$

then, applying the result from the one-dimensional case to each of the functions 
$h_{n}$, we have

$$
\begin{aligned}
\sum_{m, n=-\infty}^{\infty} I_{1}^{*}(m, n)^{2} & =\sum_{n} \sum_{m} I_{1}^{*}(m, n)^{2} \leq \sum_{n}\left\|h_{n}(x)\right\|_{2}^{2} \\
& =\int_{0}^{1} \sum_{n}\left|h_{n}(x)\right|^{2} d x=\int_{0}^{1}\left\|h(x, \cdot) \chi_{[0, x]}(\cdot)\right\|_{L^{2}(\cdot)}^{2} d x \\
& =\int_{x=0}^{1} \int_{y=0}^{x}|h(x, y)|^{2} d y d x \leq\|h\|_{2}^{2} .
\end{aligned}
$$

Beginning with this result, we may follow the same path as in the case of a one-dimensional action, using it to prove strong $(2,2)$ for the helical transform and double maximal helical transform on $l^{2}(\mathbb{Z} \times \mathbb{Z})$, then for the double maximal helical transform for a pair of commuting m.p.t.'s on a measure space, then for a pair of commuting continuous-parameter flows of m.p.t.'s. The analogue of the continuity property of the spectral measure of a m.p.t. (Theorem 2 of [1], a strengthening of Gaposhkin's necessary and sufficient condition for the Pointwise Ergodic Theorem), would follow as well. The extensions to actions of $\mathbb{Z}^{d}$ or $\mathbb{R}^{d}$ for any $d>1$ can be achieved the same way. Curiously, we do not see how to deduce the later results in this chain from their one-dimensional versions directly, without following more or less this route: for example, the sector trick does not seem to work well on the helical transform for the action of a pair of commuting m.p.t.'s.

The main use to which the helical transform was put in [1] was to prove that the spectral measure $E$ of a m.p.t. has a continuity property at 0 :

$$
\text { for any sequence } \varepsilon_{k} \rightarrow 0^{+} \text {and } f \in L^{2}, \quad E\left(0, \varepsilon_{k}\right) f(x) \rightarrow 0 \text { a.e. }
$$

This amounted to a strengthening, for m.p.t.'s, of Gaposhkin's necessary and sufficient condition for normal operators to satisfy the Pointwise Ergodic Theorem. Now that the double maximal inequality has been extended to the helical transform for flows, we can show that the spectral measure of the flow has a similar continuity property at $\infty$, amounting to a strengthening, in the measure-preserving case, of Gaposhkin's necessary and sufficient condition for the Local Ergodic Theorem [6].

9. Theorem (Continuity at $\infty$ for the spectral measure of a measure-preserving flow). For a unitary flow $\left\{U_{s}:-\infty<s<\infty\right\}$ on $L^{2}(X, \mathscr{B}, \mu)$ with spectral measure $E$ (a projection-valued measure defined for Borel subsets of $\mathbb{R}$ ), $f \in$ $L^{2}(X, \mathscr{B}, \mu)$, and any sequence $r_{k} \rightarrow \infty$, the properties (1) and (2) below are equivalent; if $\left\{U_{s}\right\}$ comes from a measure-preserving flow on $X$, then both properties hold:

(1) $E\left(-r_{k}, 0\right) f(x) \rightarrow E(-\infty, 0) f(x)$ a.e.

(2) If

$$
F_{\theta} f(x)=\lim _{n \rightarrow \infty} \int_{\frac{1}{n} \leq|s| \leq n} \frac{e^{2 \pi i s \theta} U_{s} f(x)}{s} d s \text { and } F f=F_{0} f,
$$

then

$$
F_{r_{k}} f(x) \rightarrow F f(x)+i \pi E\{0\} f(x)+2 i \pi E(-\infty, 0) f(x) \text { a.e. }
$$


Proof. Because

we have

$$
\lim _{n \rightarrow \infty} \int_{\frac{1}{n} \leq|u| \leq n} \frac{e^{2 \pi i u \lambda}}{u} d u=\pi i \operatorname{sgn} \lambda
$$

$$
\begin{aligned}
F_{r} & =\int_{-\infty}^{\infty} \lim _{n \rightarrow \infty} \int_{\frac{1}{n} \leq|u| \leq n} \frac{e^{2 \pi i u r} e^{2 \pi i u \lambda}}{u} d u d E(\lambda) \\
& =\int_{-\infty}^{\infty} i \pi \operatorname{sgn}(\lambda+r) d E(\lambda)
\end{aligned}
$$

and hence

$$
\begin{aligned}
\frac{1}{i}\left[F_{r}-F\right] & =\pi \int_{-\infty}^{\infty}[\operatorname{sgn}(\lambda+r)-\operatorname{sgn}(\lambda)] d E(\lambda) \\
& =2 \pi E(-r, 0)+\pi(E\{0\}+E\{-r\}) .
\end{aligned}
$$

From this equation the equivalence of statements (1) and (2) for any unitary flow is clear. Moreover, statement (1) clearly holds for any $f$ in

$$
\bigcup_{K=1}^{\infty} E(-K, K) L^{2}(X, \mathscr{B}, \mu),
$$

a dense set in $L^{2}(X, \mathscr{B}, \mu)$. Since in the case of a measure-preserving flow we have a maximal inequality for this family of operators (Theorem 6), in this case both (1) and (2) hold for all $f \in L^{2}(X, \mathscr{B}, \mu)$.

We say that the Wiener-Wintner property holds for a family of a.e. convergent sequences of functions if there is a single set of measure 0 off of which each sequence in the family converges pointwise. The interest in this property arises when the family is indexed by a parameter running through an uncountable set. This property has facilitated the application of the Pointwise Ergodic Theorem to the spectral analysis of stationary processes, and we hope that it may do likewise for the helical transform. At this point, however, we are only able to note that the property holds for the Local Ergodic Theorem. In order to avoid having to distinguish between sets of measure 0 and inessential sets (subsets of sets of measure 0 ), we will assume in this section that the measure spaces under consideration are complete.

10. Proposition (Wiener-Wintner property for the Local Ergodic Theorem). Let $\left\{T_{s}:-\infty<s<\infty\right\}$ be a measure-preserving flow on a complete measure space $(X, \mathscr{B}, \mu)$ and $f \in L^{1}(X, \mathscr{B}, \mu)$. Then there is a set $N \subset X$ with $\mu(N)=0$ such that for all $\theta \in \mathbb{R}$ and $x \notin N$,

$$
\lim _{t \rightarrow 0^{+}} \frac{1}{t} \int_{0}^{t} e^{2 \pi i s \theta} f\left(T_{s} x\right) d s=f(x) .
$$

Proof. Again the required maximal inequality is already available-it is just the Maximal Ergodic Theorem for measure-preserving flows-so we only need to check convergence on a dense set. Even though the handling of these sets of measure 0 is somewhat delicate, and indeed some of the sets which must be considered cannot immediately be seen to be measurable, still in this case Banach's Principle can be applied successfully. Such an approach may be instructive if one has in mind eventually to try to prove analogous theorems for 
more complicated operators, but there is also an easy direct proof (we thank the referee for this observation): we may write

$$
\frac{1}{t} \int_{0}^{t} e^{2 \pi i s \theta} f\left(T_{s} x\right) d s=\frac{1}{t} \int_{0}^{t} f\left(T_{s} x\right) d s+\frac{1}{t} \int_{0}^{t}\left(e^{2 \pi i s \theta}-1\right) f\left(T_{s} x\right) d s,
$$

and bound the second term by a constant times $|\theta| t f^{*}(x)$ (where $f^{*}$ is the usual ergodic maximal function), and then easily take the limits as $t \rightarrow 0$.

11. Remark (Failure of weak $(1,1)$ for the double maximal helical transform). $H^{* *} f(x)$ (see definition above in $(6)$ ) does not satisfy a weak $(1,1)$ maximal estimate for $f \in L^{1}(X, \mathscr{B}, \mu)$. We know of two ways to see this:

(11.1) M. Lacey and M. Marcus (personal communication) have provided an example of an independent identically-distributed sequence $\left\{X_{k}\right\}$ for which weak $(1,1)$ fails. Using results of [9], they show that we can have $X_{k} \in L^{1}$ with

$$
P\left\{\left|X_{k}\right|>\lambda\right\} \sim\left(\lambda \log \lambda \log \log ^{1+\beta} \lambda\right)^{-1}, \quad 0<\beta \leq 1,
$$

but with the associated double helical maximal function $H^{* *}$ equal to infinity almost everywhere.

(11.2) Kolmogorov's example of a divergent Fourier series for an $L^{1}$ function may also be transferred to this setting. For if the operator $H^{* *}$ were weak $(1,1)$, then by arguments similar to ones used above (for example using time $\delta$ maps) we could conclude that the analogous operator $F^{* *}$ for continuousparameter flows (see (6)) is also weak $(1,1)$, and in particular this would hold true for the translation flow on $\mathbb{R}$. Because of the well-known formula (see [7])

$S_{n} f(x)=\frac{1}{2 \pi i}\left[e^{2 \pi i n x} \int_{0}^{1} \frac{e^{-2 \pi i n t} f(t)}{x-t} d t-e^{-2 \pi i n x} \int_{0}^{1} \frac{e^{2 \pi i n t} f(t)}{x-t} d t\right]+K_{n} * f(x)$,

where $K_{n} \in L^{\infty}[0,1)$, for the $n$th partial sum $S_{n} f(x)$ of the Fourier series of an integrable function $f$ on $[0,1)$, this would imply that $S_{n}$ is also weak $(1,1)$, an absurdity in view of Kolmogorov's example of an $f \in L^{1}$ for which $\sup _{n}\left|S_{n} f(x)\right|=\infty$ a.e.

12. Remark (The case $p \neq 2$ ).

(12.1) For $p>2$, the analogue of statement (3) continues to hold, since

$$
\left\|I^{*} h\right\|_{l^{p}(\mathbb{Z})} \leq\left\|I^{*} h\right\|_{l^{2}(\mathbb{Z})} \leq C\|h\|_{L^{2}[0,1)} \leq C\|h\|_{L^{p}[0,1)} .
$$

(12.2) For $1<p<2$, the analogue of statement (3) no longer holds for all $h$, since if it did we would have the chain of inequalities

$$
\|h\|_{L^{2}[0,1)} \leq C\left\|I^{*} h\right\|_{l^{2}(\mathbb{Z})} \leq C\left\|I^{*} h\right\|_{l^{p}(\mathbb{Z})} \leq C\|h\|_{L^{p}[0,1)},
$$

the last member of which does not always dominate the first member.

(12.3) The analogue of statement (4) (the strong $(p, p)$ estimate for the maximal helical transform on $l^{p}$ ), however, does hold for all $p, 1<p<$ $\infty$. This can be seen as follows. By the Carleson-Hunt Theorem, if $f \in$ $L^{p}[0,1), p>1$, then also

$$
\sup _{n}\left|\int_{0}^{1} \frac{e^{2 \pi i n t} f(t)}{x-t} d t\right| \in L^{p}[0,1) .
$$


This implies immediately that if $f \in L^{p}(\mathbb{R}), p>1$, then

$$
\sup _{n}\left|\int_{-\infty}^{\infty} \frac{e^{2 \pi i n t} f(t)}{x-t} d t\right| \in L^{p}(\mathbb{R})
$$

(subdivide $\mathbb{R}$ into intervals of length 1 , apply Carleson-Hunt on each interval, and sum). To obtain a similar estimate when the supremum is taken over all $\theta \in \mathbb{R}$ rather than just over $n \in \mathbb{Z}$, we show (by change of variable) that therefore

$$
\sup _{k \in \mathbb{Z}}\left|\int_{-\infty}^{\infty} \frac{e^{2 \pi i \frac{k}{2^{n}} t} f(t)}{x-t} d t\right|
$$

has $L^{p}$ norm bounded by a constant times the $L^{p}$ norm of $f$, with the constant independent of $n$. The method of [8] then applies to deduce the discrete maximal inequality from its continuous analogue.

This approach could be used to establish many of the results considered above, but Remark 12.2 shows that some of the assertions involving $I^{*}$ cannot be proved in this manner. Curiously, the maximal operator for partial Fourier coefficients in some cases behaves differently than the maximal operator for partial sums of Fourier series.

\section{REFERENCES}

1. J. Campbell and K. Petersen, The spectral measure and Hilbert transform of a measurepreserving transformation, Trans. Amer. Math. Soc. 313 (1989), 121-129.

2. A. P. Calderón, Ergodic theory and translation invariant operators, Proc. Nat. Acad. Sci. U.S.A. 59 (1968), 349-353.

3. L. Carleson, On convergence and growth of partial sums of Fourier series, Acta Math. 116 (1966), 135-157.

4. H. Davenport and H. Halberstam, The values of a trigonometrical polynomial at well spaced points, Mathematika 13 (1966), 91-96.

5. C. Fefferman, On the convergence of multiple Fourier series, Bull. Amer. Math. Soc. 77 (1971), 744-745.

6. V. F. Gaposhkin, The Local Ergodic Theorem for groups of unitary operators and second order stationary processes, Math. USSR-Sb. 39 (1981), 227-242.

7. R. Hunt, On the convergence of Fourier series, Orthogonal Expansions and their Continuous Analogues, (D. T. Haimo, ed.), Southern Illinois Univ. Press, Carbondale, 1968, pp. 235255.

8. R. Hunt, B. Muckenhoupt, and R. Wheeden, Weighted norm inequalities for the conjugate function and Hilbert transform, Trans. Amer. Math. Soc. 176 (1973), 227-251.

9. M. B. Marcus, $\xi$-radial processes and random Fourier series, Mem. Amer. Math. Soc., no. $181,1987$.

10. K. Petersen, Almost everywhere convergence of some nonhomogeneous averages, Proc. Internat. Conf. Almost Everywhere Convergence in Probability and Ergodic Theory (Columbus, Ohio, 1988), Academic Press, 1989, pp. 349-367.

11. Elias M. Stein, Singular integrals and differentiability properties of functions, Princeton Univ. Press, Princeton, N. J., 1970.

12. Alberto Torchinsky, Real-variable methods in harmonic analysis, Academic Press, New York, 1986.

13. N. Wiener, The ergodic theorem, Duke Math. J. 5 (1939), 1-18.

Department of Mathematics, CB\#3250, Phillips Hall, University of North Carolina, Chapel Hill, North Carolina 27599 